\title{
Assessment of Solid Waste Management in Residential Townships in Lusaka Zambia
}

\author{
Allan Zulu* \\ MSc Public Health, PhD Public Health, Zambia \\ *Corresponding Author: Allan Zulu, MSc Public Health, PhD Public Health, Zambia.
}

Received: October 14, 2019; Published: October 18, 2019

DOI: 10.31080/ASAG.2019.03.0685

\begin{abstract}
This study assessed practices, methods and perceptions of solid waste management (SWM) in selected townships in Lusaka Zambia. The aim was to establish effectiveness of SWM in order to have a basis for advocating for measures to address issues related to improved solid waste management. Specifically, the research aimed to determine the socio-demographic and economic profiles of people, methods and practices of solid waste disposal and to obtain people's perception on solid waste management. The study targeted 120 respondents purposely selected from low, medium and high-density townships of Misisi, Kabwata and Ibex Hills in Lusaka, Zambia. The study collected data by use of a self-administered questionnaire through random sampling and was analysed through SPSS version 23. The findings of this study revealed that solid waste management was a challenge in Lusaka, Zambia. The majority of respondents (37.5\%) stated that they generated all kinds of waste from their households and $51.6 \%$ stated that they had never heard of appropriate solid waste management. Of those that emptied waste in public waste bins, $35 \%$ stated that they walked 5-10 minutes to access public waste bins, 19.2\% stated that public bins, which emptied weekly, were small and ever full. Fifty-two (52\%) percent of respondents stated that there was a problem with solid waste disposal and $56.7 \%$ stated that solid waste disposal contributed to diseases in their area. This study concluded that there were challenges of solid waste management in terms of practices and methods in selected areas of Lusaka, Zambia.
\end{abstract}

Keywords: Solid Waste; Waste Management; Household Waste; Disposal; Assessment

\section{Introduction}

Solid Waste Management (SWM) is one of the major public health concerns worldwide. The problem is more pronounced in Less Developed Countries (LDCs) of which Zambia is one of them. Poor management of waste not only leads to declining environmental health conditions but contributes to disease outbreaks as well. Studies have indicated that solid waste management is a problem in many developing countries especially in high-density areas [1,2]. Effluent and other types of solid waste are dumped either in rivers or in drainages resulting into water and air pollution. Inadequate sanitary facilities and transport to collect waste in LDCs greatly contributes to pollution levels [3].
Solid waste is found in every place where there is human activity. Accumulated materials as a result of human activity, whether from domestic or industrial operations that are deemed unworthy or rejected, require to be disposed of [4]. In the United States of America (USA) in spring and summer of 1987 there was a problem of solid waste disposal as the garbage barge could not find a place to dispose the garbage [2]. About 254 tons of garbage which include durable and nondurable goods, containers and packaging, food scraps, yard trimmings and other miscellaneous inorganic waste from residential, domestic and industrial sources is disposed of every year by Americans [2]. In most countries, garbage collection is the responsibility of the local government since the late $19^{\text {th }}$ century as at that time, it was recognized that, because of garbage, 
rats and flies that carry diseases such as plague and typhus were attracted by it. This required therefore that garbage be disposed of outside the cities [2]. Activities such as economic development, urbanization and improved standards of living, contribute to accumulation of Municipal Solid Waste (MSW) resulting into environmental degradation leading to public health issues (Central Pollution Control Board [CPCB], 2000). This has resulted into higher levels of water and air pollution in cities. According to the United Nations Centre for Human Settlement [3], pollution levels are worse in LDCs because of other contributing factors such as transport, infrastructure and inadequate sanitation facilities. The problem of waste disposal has reached a greater proportional in LDCs including Zambia. In Zambia, the rate at which waste is generated is high especially in high-density townships. The high population contributes to the fast rate at which waste is generated. Other compounding factors include insufficient capacity and equipment for waste collection and disposal. Inadequate recovery and non-recycling of wastes such as inorganic materials and inadequate awareness on sound waste management on how they impact on the environment and health are other contributing factors [5].

\section{Study objectives aim}

The aim of this study was to determine practices and methods of SWM and to get an overview of the perception of people on solid waste management in selected areas of Lusaka Zambia.

\section{Objectives}

The main objective of the study was to establish the effectiveness of solid waste management in order to have a basis for advocating for measures to address issues related to improved solid waste management.

\section{Specifically the study}

- $\quad$ Assessed socio-demographic and economic profiles of people

- $\quad$ Determined common waste management practices

- $\quad$ Determined methods of solid waste management in selected areas of Lusaka, Zambia.

\section{Review of the Literature}

Literature review reveals that there has been a lot of information of researched work on this topic. There has been information on different methods of waste disposal, the high rate of wastes generated but not much on strategies to improve waste disposal. There is need to come up with strategies on how to improve waste disposal especially in Lusaka Zambia. Examining waste disposal at household and community levels, we find that evidence is mixed on waste management. Until the 1970 s, people did not pay attention to what was done with the garbage after it was collected from their residences. Waste was either disposed in the dumps or burnt or poured into nearby rivers, lakes or oceans [2]. Schneider also stated that methods of disposal were outlawed in many states as it merely transferred garbage from one place to the other. Other waste management disposal problems have been associated with odours, pests and spills.

A study conducted in Saudi Arabia on industrial effluent quality, pollution monitoring and environment management revealed that there has been a reduction in the pollution of underground water after implementing several strategies to implement the zero discharge policy [1]. Uncontrolled wastewater contributes to water resource degradation, which in turn affects agriculture production and has an impact on public health [1]. On the other hand, if wastewater is treated the opposite is the truth; treated wastewater creates water sources which can be used for agriculture irrigation and improve underground water sources by recharging aquifers [1].

Another study on the pollution sources and mortality rates across rural-urban areas in the United States revealed that in areas where there was coal mining, there was a high total number of cancer and respiratory disease mortality rates [6]. A pollution prevention and community environmental health study by Hendryx, Fedorko and Halverson [6], concluded that community pollution prevention plays an integral role in assisting local governments by proactively mitigating toxic emissions.

A study was conducted in Zambia to investigate practice and perceptions on solid waste management with the possibility of establishing a site waste management plan in the Zambian construction Industry. The study involved Lusaka and Copperbelt provinces, which were considered to have a high concentration of industries and construction works. The study collected data through surveys, interviews and questionnaires. The results of this study indicated that the most common method of waste management was disposal [7]. The study concluded that construction industry has poor practice of waste management, that this method of waste management is not environmental friendly and that it is important to adopt a better plan that can be an effective way of addressing challenges of waste management [7]. 
A research was conducted to investigate services provided to markets in Lusaka by Lusaka City Council (LCC) in order to determine the quality of services of garbage collection. The study involved 264 participants who were selected using simple random sampling. The study used both primary and secondary data and was analyzed by both qualitative and quantitative methods. The study revealed that LCC had inadequate capacity to manage solid waste in the selected markets. However, some of the marketeers expressed satisfaction in the way garbage was managed. The study also found out that LCC had difficulties with garbage collection from secondary points due to inadequate tools. The study recommended that LCC should put in place garbage collection equipment in order to effectively manage garbage in the markets.

\section{Rationale}

The issue of waste management was identified as worth investigating because of its impact on public health. The study addresses the effectiveness of prevention of public health issues such as Cholera and other waterborne diseases and air pollution, which have become common in Zambia. It highlights among others, factors leading to the spread of diseases in various demographic location of the country. The significance of this study is that the findings will be beneficial to the communities that have high prevalence rate of diarrheal diseases and to the country as a whole.

The research will also guide policy makers and other stakeholders involved in waste disposal management and disease prevention to improve and change existing strategies for waste disposal management. The research findings will help reshape the implementation programs that have to do with waste disposal management, risk reduction strategies and improvement of health of affected people throughout the country. It will also create awareness on the relationship between the environment and public health. This study specifically addressed the following statements:

- The correlation between solid waste management practices and public health issuesin selected townships in Lusaka Zambia

- $\quad$ Effects of inappropriate solid waste management practice and environmental health conditions

- $\quad$ Assessed the level of solid waste management problems in selected townships in Lusaka Zambia.

\section{Methodology}

Study design and data collection

The study employed a non-intervention exploratory study method. A sample size of 120 respondents were purposely selected in low, medium and high residential townships of Lusaka Zambia in Misisi, Kabwata and Ibex Hill townships. The study employed a semi-structured questionnaire to collect data from respondents, which included both male, and female of above 18 years of age who were heads of households. The study excluded all respondents below 18 years and dependents who were above 18 years of age. The researcher clearly explained to all respondents that the questionnaire was administered for research purposes and that there were no any benefits for taking part in the research. The study employed random sampling method to cut on time and travel costs.

\section{Study site}

The study was conducted in 3 residential areas in Misisi, Kabwata and Ibex Hill townships in Lusaka, Zambia and drew respondents from purposely selected households. The objective of the selection was to provide a balanced representation from low, medium and high cost residential areas. Townships where conveniently selected for easy collection of data in that respondents were to be found in one place. This made it more efficient and cost effective for data collection.

\section{Study population}

The study population included all male and female adults of 18 years and above. Respondents below the age of 18 were excluded from the target population bearing in mind that they could not be heads of households and therefore would not fully understand the objective of responding to a semi-structured questionnaire.

\section{Data analysis}

Data was analysed by use of SPSS version 23. This study conducted univariate data analysis to examine, describe variables and to summarize numerical information in order to reveal the patterns in a more systematic way. The study also employed bivariate data analysis to correlate data in order to establish relationships between variables [8-12].

\section{Findings descriptive analysis}

Data from 120 respondents were analysed. Descriptive data showed that the majority of respondents where females $(50.8 \%)$, the age range was between 18 - 29 years (43.3\%), that the most frequent number of household members in the households was 5 at $(21.7 \%)$ and the number of households with members below the age of 15 was at $41.7 \%$. Most respondents stated that they had lived in Lusaka for more than 10 years and that the majority (50.8\%) attended education. Sixty- five percent (65\%) of respondents where professionals and $90.8 \%$ had not heard of solid waste management in Lusaka. Forty-six percent (46\%) of those who heard about solid 
waste management heard it on radio, television, posters and at school. The majority of the respondents (51.6\%) stated that they had never heard of appropriate solid waste management disposal, while $70 \%$ of respondents stated that they generated all types of solid waste including plastic bags, food waste, tins and glass from their households. And of those, 37.5\% emptied their waste containers every day while $41.7 \%$ emptied their solid waste in public containers in their areas. Of those who stated to have public waste bins in their areas, thirty-five percent (35\%) stated that they took 5- 10 minutes to reach the public waste bins and $46 \%$ stated that their waste bins were emptied once a week. Nineteen percent (19.2\%) stated that the public bins they used were small. The majority of respondents (51.7\%) stated that there was a problem with solid waste disposal in their areas, fifty- six point seven percent (56.7\%) stated that solid waste disposal contributed to diseases in their area while $33.3 \%$ stated that solid waste disposal was good in their area.

\section{Cross tabulations}

\section{Residential area}

Data analysis showed that 39\% of respondents lived in low cost residential areas. Level of education attained by a respondents had an association with the type of residential areas they came from with a p-value $=0.046$ significant at 0.05 . Occupation of respondents was distributed in a way that majority of the respondents were professional workers $(65 \%)$ of which most of them lived in medium residential area (40\%). Occupation and residential areas had an association ( $\mathrm{p}$-value $=0.001$ ) which was significant at 0.05 level. Residential areas where respondents came from was associated with the type of waste generated from a particular household (p-value 0.013 ) at 0.05 significant level.

Disposal of waste was associated with residential areas of participants at ( $p$-value $=0.00$ ) which was significant at 0.05 . Majority of respondents who stated not having public bins near their residential areas where from medium residential areas (56\%) at $\mathrm{p}$-value $=0.00$. How long it took to get to the public bin was not applicable to most of the respondents (60\%), but this response was associated with residential areas a respondent was from $(\mathrm{p}$-value $=$ 0.00 ) at 0.05 significant level. If a respondent had a public bin near home, how often it was emptied was not applicable to most of the respondents (50\%) and this was associated with residential areas from ( $\mathrm{p}$-value $=0.00)$ at 0.05 significant level. Describing a public bin was not applicable to most of the respondents (50\%) and this was associated with residential areas from $(p$-value $=0.00)$ at 0.05 significant level. Most of the respondents stated that disposal of waste was not a problem in their residential areas of which most of them were from medium residential areas. This response was associated with respondents' residential area $(p$-value $=0.00)$ at 0.05 significant level. Most of the respondents stated that solid waste disposal contributed to diseases in their area (57\%) of which most of them were from low residential areas (47\%) the association between residential areas and if solid waste contributed to diseases was significant at $0.05(\mathrm{p}$-value $=0.00)$. Respondents were asked to evaluate the state of solid waste disposal in their area and the majority (33\%) said that it was good of which most of them were from medium residential areas (53\%) this evaluation was associated with which residential areas respondents were format 0.05 significant level with $\mathrm{p}$-value $=0.00$.

\section{Level of education}

Respondents level of education attained was significantly associated with age range at 0.05 significant level with $\mathrm{p}$-value $=0.00$. The majority of respondents (91\%) stated that they had never heard of appropriate solid waste management regardless of the level of education. Disposal of waste and education level were associated ( $\mathrm{p}$-value $=0.003$ ) at 0.05 significant level. However, there was an association between educational level and how often the public bin was emptied ( $p$ - value $=0.033$ ) at 0.05 significant level.

\section{Gender}

The majority of respondents (51\%) who stated that solid waste contributed to diseases were female. This statement was significant at ( $\mathrm{p}$-value $=0.045$ ) significant at 0.05 level.

\section{Age range}

The kind of waste disposed was associated with age group of the respondents ( $\mathrm{p}$-value $=0.002$ ) at 0.05 significant level. Plastic (polythene) bag was the most common type of container used to collect solid waste by $37 \%$ of the respondents of which $57 \%$ were between 18 and 29 years old. Age group was associated with how often respondents disposed their waste $(p$-value $=0.008)$ at 0.05 significant level.

\section{Occupation}

Occupation had an association with the type of container used as means of waste disposal by respondents $(p$-value $=0.025)$ at 0.05 significant level. Most of the respondents (38\%) emptied their solid waste on a daily basis and $69 \%$ of these responses were from professional workers. The majority of respondents (42\%) disposed their collected waste in public bins and $50 \%$ of these were 
professional workers. How respondents disposed their collected waste was dependent on their occupation $(p$-value $=0.01)$ at 0.05 significant level.

\section{Significance and Conclusion}

This research assessed socio-demographic and economic profiles of people, determined common waste management practices and methods of solid waste management in selected areas of Lusaka, Zambia. The study showed that $91 \%$ had not heard of solid waste management and had never heard of appropriate solid waste management disposal. Seventy percent (70\%) of respondents stated that they generated all types of solid waste including plastic bags, food waste, tins and glass from their households. The majority also stated that they took a 5 - 10 minutes' walk to empty their bins to public bins and that the public bins were small and ever full. The majority of respondents (51.7\%) stated that there was a problem with solid waste disposal in their areas and that solid waste disposal contributed to diseases in their area. The study therefore concluded that there was inadequate knowledge of solid waste management and poor practices of appropriate waste disposal among all respondents regardless of age, sex and level of education. The study also concluded that inappropriate waste disposal contributed to diseases.

The findings of the study will assist policymakers to come up with mitigation strategies on how to improve waste management disposal. It will also assist to understand the relationship between inappropriate waste management and public health concerns.

\section{Bibliography}

1. Ahmad M., et al. "Industrial effluent quality, pollution monitoring and environmental management". Environment, Monitoring and Assessment 147 (2008): 297-306.

2. Schneider MJ. Introduction to Public Health. (3rd ed). Jones and Bartlett Publishers. London (2011).

3. United Nations Center for Human Settlements (Habitat). The state of the world's cities 2001. Nairobi, Kenya (2001).

4. Sridhar MKC. Wastes-Their nature and impact on environment. Paper presented at a training workshop on planning and management of waste systems and environmental care, Ibadan, Nigeria (1998).

5. http://www.un.org/esa/dsd/resources/res_pdfs/csd18/06may/waste/Zambia.pdf
6. Hendryx M., et al. "Pollution Sources and Mortality Rates Across Rural-Urban Areas in the United States". The Journal of Rural Health 26 (2010): 383-391_c.

7. Muleya F and Muyoba R. "Minimizing Construction Material Waste through Design". National Council for Construction, Construction News Magazine, Zambia (2010).

8. Neuman wL. Social Research Methods. Qualitative and Quantitative Approaches (4th ed) Needham Heights MA 02494 Allyn and Bacon (2000).

9. Ana GREE., et al. "Solid Waste Management Problems in Secondary Schools in Ibadan, Nigeria". Journal of Environmental Health 74 (2011).

10. Bharadwaj L., et al. "Waste disposal in First-Nations communities: the issues and steps toward the future". Journal of Environmental Health 68.7 (2006).

11. Lermen HS. "Environmental perception as a public health issue in a socially vulnerable area of Brazil" (2006).

12. Murti M., et al. "Public Health Response to Striking Solid Waste Management". Journal of Environmental Health 74 (2011): 2226.

Volume 3 Issue 11 November 2019 (C) All rights are reserved by Allan Zulu. 